\title{
Oferta y demanda de los guías de turismo de la Zona 3- Ecuador
}

\author{
Johanna Monge-Martínez', Noemy Gaviño- Ortíz², Carmen Vaca- Vaca ${ }^{3}$, Javier Salazar - Mera ${ }^{4}$ \\ Fecha de recepción: 25 de Abril 2018 \\ Fecha de aceptación: 22 de Junio 2018
}

\section{Resumen}

Los guías de turismo establecen el primer vínculo con el turista. La calidad del servicio determina la imagen del destino y grado de satisfacción del visitante. Surge la necesidad de profesionales competentes con amplios conocimientos actualizados. El documento tiene como objetivo analizar la perspectiva oferta - demanda de los guías de turismo de la zona tres del Ecuador, conformada por las provincias de Chimborazo, Cotopaxi, Pastaza y Tungurahua. La metodología incluyó investigación de campo, un cuestionario estructurado; aplicado a los guías y representantes de operadoras turísticas del contexto. Los resultados indican que los guías turísticos deben tener una formación específica.

Palabras clave: Formación, guías, turismo.

\section{Abstract}

Tourist guides establish the first link with the tourist. The quality of the service determines the image of the destination and the degree of satisfaction of the visitor. The need arises for competent professionals with extensive up-to-date knowledge. The objective of this document is to analyze the offer - demand perspective of the tourist guides of zone three of Ecuador, formed by the provinces of Chimborazo, Cotopaxi, Pastaza and Tungurahua. The methodology included field research, a structured questionnaire; applied to guides and representatives of tour operators in the context. The results indicate that tour guides must have specific training.

Keywords: Training, guides, tourism.

\section{Introducción}

La recreación ha estado ligada al hombre desde su misma concepción. Huizinga (2007) afirma a través de su estudio que existen dos actividades innatas del ser humano Homo Faber que equivale al hombre que trabaja y Homo Ludens al hombre que juega, es esta última donde el turismo se posiciona como una alternativa primordial para los viajeros. La actividad turística es la consecuencia de un fenómeno social cuyo punto de partida radica en la existencia del tiempo libre (Boullón, 2006). En esta consideración, el turismo se transforma en un abanico de posibilidades y alternativas que dan paso a la gran dinámica de mercado entre la oferta y demanda turística. El turismo no solo es visto como un medio de recreación o aprovechamiento del tiempo de ocio, sino también, como una gran herramienta para el fortalecimiento de la interculturalidad y el desarrollo económico. Durante las seis últimas décadas, el turismo ha experimentado una continua expansión y diversificación, convirtiéndose en uno de los sectores económicos de mayor envergadura y crecimiento del mundo (OMT, 2017). A escala mundial los arribos de turistas internacionales han pasado de 25 millones en 1950 a 1.186 millones en 2015. De forma análoga, los ingresos por turismo internacional obtenidos por los destinos de todo el mundo han pasado de 2.000 millones de dólares en 1950 a 1'260.000 millones en 2015 (OMT, 2016). En el 2017 el turismo internacional correspondía al 7\% de las exportaciones mundiales de bienes y servicios, frente al $6 \%$ obtenido en el 2014. Como categoría mundial de exportación, ocupa el tercer puesto, tan solo por detrás de combustibles y de productos químicos, y por delante de alimentación y de la industria de automoción. En muchos países en desarrollo, el turismo es incluso el primer sector en ex- portaciones (OMT, 2017). América, Asia y el Pacífico registraron un crecimiento de llegadas de turistas internacionales cercano al 6\% (OMT, 2017). En Ecuador el movimiento turístico también va en aumento, a país llegan miles de turistas extranjeros por eventos, congresos, negocios o en búsqueda de nuevos destinos por explorar. Según informa el Ministerio de Turismo del Ecuador, a través de su página web, en el año 2017 arribaron un total de 1.617.914 visitantes lo que representa un crecimiento de 14\% con respecto al 2016 (MINTUR, 2017a). Entre 2015 y 2016 más de 30 proyectos de inversión turística se encontraban en construcción en Ecuador, con una inversión de USD 750 millones. Para el 2017 se estimó una inversión de 1.600 millones, provenientes de capitales nacionales y extranjeros. El turismo representa el 6,4\% del total de empleados en la economía, lo que permite que esta actividad se ubique entre las 6 industrias económicas de mayor contribución al empleo nacional (INEC, 2017)

En cuanto al turismo interno existen 12.3 millones de desplazamientos registrados durante el 2016. El 40\% de ellos se realizó durante los diferentes feriados, esto representó más de 4.9 millones de viajes. Todo este movimiento interno dinamizó la economía nacional con 285.5 millones de dólares (MINTUR, 2017b). El Ministerio de Turismo trabaja para que la actividad turística sea el eje fundamental del cambio de la matriz productiva (MINTUR, 2017c). Las políticas públicas están actuando para centrar esfuerzos e impulsar el turismo. Por tanto, la industria turística debe ofrecer al viajero diferentes experiencias y emociones de forma sostenible y de calidad, esto determina el nivel de satisfacción del turista. Sin duda el desarrollo de esta actividad, está directamente relacionada con la labor desempeñada por los profesionales del sector y es aquí donde el componente humano juega un papel fundamental para el desarrollo del turismo (Bañuls, Rodríguez, \& Jiménez, 2007).

El desarrollo del talento humano en sus diferentes formas, ya sea de formación, capacitación o adiestramiento son vitales para el crecimiento de la actividad turística en cualquier lugar. Son los guías de turismo quienes están en directo contacto con el turista, por tanto son los principales abanderados del turismo en un destino (Rebolledo \& Fica, 2010). Cuando los turistas viajan a un destino esperan conocer parte de la historia, la cultura, las tradiciones y las bellezas arqueológicas, reflejadas en todas partes, incluso en el servicio que ofrecen los guías (Zárraga, Corona, \& Molina, 2010). Es posible afirmar entonces, que la empresa turística se destaca por la importancia del rol en la personalización del servicio, es decir, la relación persona-persona. Los guías turísticos son los encargados de algunas responsabilidades como proveedores de experiencias turísticas (Cohen 1985; Pond 1993). Por ejemplo, en el texto que hace relación a la regulación del guía de aventura AENOR (2016), manifiesta las normas de seguridad ISO para el turismo de aventura (ISO 21101 y TR 21102) y se explica una gama de aspectos que debe desarrollar dicho profesional como: competencia técnica, servicio al cliente y manejo de grupos, interpretación de la historia natural y cultural; entre otros. A su vez en un estudio realizado por Ham \& Weiler (2003), sobre capacitación para guías turísticos en Latinoamérica se concluye que los resultados obtenidos a largo plazo generan efectos multiplicadores. Los guías de turismo son los que toman la responsabilidad de proveer alta calidad de interpretación sobre recursos naturales y culturales. Su misión es hilar los servicios que recibirá el visitante como un verdadero tejido, por medio del cual, el cliente irá recibiendo

'Master en Dirección y Planificación del Turismo. Profesora Universitaria. Facultad de Ciencias Humanas y de la Educación. Universidad Técnica de Ambato. UTA. Ambato-Ecuador. E-Mail: jg.monge@uta.edu.ec ${ }^{2}$ Magister en Administración de Empresas mención Planeación. Profesora Universitaria. Facultad de Ciencias Humanas y de la Educación. Universidad Técnica de Ambato. UTA. Ambato- Ecuador. E-Mail: noemyhgavinioo@uta.edu.ec

${ }^{3}$ Magister en Turismo Sostenible y Desarrollo Local. Profesora Universitaria. Facultad de Ciencias Humanas y de la Educación. Universidad Técnica de Ambato. UTA. Ambato- Ecuador. E- Mail: carmenivacav@uta.edu.ec

${ }^{4}$ Magister en Tecnología de Información y Multimedia Educativa. Profesor Universitario. Facultad de Ciencias Humanas y de la Educación. Universidad Técnica de Ambato. UTA. Ambato- Ecuador. E-Mail: javiers@uta.edu.ec 
las experiencias que se han programado para él, sin sobresaltos y con credibilidad en el gran mercado turístico local, regional, nacional e internacional (Prado, 2001). Así mismo, los empleadores esperan que el guía proporcione alta calidad en el servicio, para maximizar no solo la satisfacción del visitante sino además los márgenes lucrativos de la actividad (Cohen 1985; Pond 1993). En este sentido Bote (1995), asegura que la formación en turismo debe constituir un eje fundamental del modelo de desarrollo cualitativo propugnado desde el ámbito académico y que permita otorgar calidad al servicio. Podemos asegurar entonces que existe una relación directa entre formación en turismo y calidad de la experiencia turística (Rebolledo \& Fica, 2010).

El perfil profesional del guía de turismo tiene la necesidad de apoyarse en la alta formación académica por competencias. Las competencias se conciben como la capacidad de movilizar recursos cognitivos para hacer frente a un tipo de situaciones o contextos mediante la práctica (Perrenound 2008 citado por López 2013), existen tres competencias laborales que fomentan una relación más positiva entre el guía de turista y el turista logrando romper todas las barreras interculturales. Los factores son: conocimiento, actitud y habilidades (Swarbrooke, 1999). Estas competencias desarrollan el valor estratégico del capital humano como capacidad distintiva y conducen a plantear la necesidad de diseñar políticas de educación y formación de calidad en turismo (Bañuls, Rodríguez, \& Jiménez, 2007). Un excelente guía en un destino eleva el nivel de la industria, mientras que un guía menos competente puede perjudicar el negocio con la misma facilidad (AENOR, 2016). En razón de las consideraciones anteriores, la formación del guía turístico es vital (Prado, 2001).

El Ecuador está dividido por zonas administrativas, la zona tres está conformada por las provincias de Chimborazo, Cotopaxi, Pastaza y Tungurahua. Los turistas se sienten atraídos por visitar estas provincias debido a los atractivos con los que cuentan. En el listado de los principales destinos del Ecuador para turismo receptivo en el año 2017 el Parque Nacional Cotopaxi (Cotopaxi) ocupó el séptimo lugar, Baños de Agua Santa (Tungurahua) ocupó el puesto once y la Reserva de Producción de Fauna Chimborazo (Chimborazo) ocupó el puesto dieciocho (MINTUR, 2017d), sin embargo el estado cuenta con otros destinos turisticos de talla internacional como son Quito, la capital del Ecuador, las Islas Galápagos, la Región Amazónica, entre otros. La ejecución de tours está a cargo de las agencias de viajes operadoras legalmente autorizadas para desarrollar dicha actividad, las mismas que de acuerdo al artículo 121 del Reglamento General de Actividades Turísticas (2011), tienen la obligación de contratar a un guía profesional de turismo por cada quince pasajeros. Esta investigación tiene como objetivo analizar la perspectiva de la oferta y la demanda de los guías de turismo de la zona tres del Ecuador.

\section{Metodología}

Para estudiar la oferta y demanda de los guías de turismo en la zona tres, conformada por las provincias y sus cantones objeto de estudio así: Chimborazo (cantón Riobamba), Cotopaxi (cantón Latacunga), Pastaza (cantón Puyo) y Tungurahua (cantón Baños); se empleó una investigación de tipo exploratoria, por la escaza información que existe sobre el tema y la dificultad que representa la recolección de datos en las operadoras turísticas y a los guías de turismo que no se les encuentra en un solo lugar; además, es una investigación descriptiva y explicativa, tratando de conocer la estructura de las operadoras y el comportamiento laboral de los guías; la metodología utilizada fue la investigación de campo. Se aplicó dos cuestionarios estructurados de cuatro preguntas cada uno, con preguntas de selección múltiple. La población total fue 108 personas, se dividió en 58 representantes de las agencias de viajes operadoras de turismo. y 50 guías. Los guías de turismo según el Reglamento de Guianza Turística del Ecuador (2016) se clasifican en guía local, guía nacional y guía nacional especializado. La población de estudio fue seleccionada de acuerdo a los cantones más turísticos de cada provincia.

\section{Resultados}

La población investigada se concentra mayoritariamente en la ciudad de Baños por ser un referente turístico tanto nacional como internacional. Este destino ofrece actividades turísticas a desarrollarse durante todo el año y está entre los 7 lugares más visitados en Ecuador. En Ambato, Riobamba, Latacunga y Puyo, capitales de provincia, se concentran las operadoras de turismo que ofertan tours para visitar los diferentes destinos turísticos del país y principalmente de la zona 3 (ver tabla 1).

Tabla 1. Operadoras de turismo de la zona 3

\begin{tabular}{|c|c|c|}
\hline \multirow{2}{*}{ Ciudad } & \multicolumn{2}{|c|}{ Operadoras } \\
\hline & $\#$ & $\%$ \\
\hline Ambato & 12 & 21 \\
\hline Riobamba & 10 & 17 \\
\hline Puyo & 6 & 10 \\
\hline Baños & 24 & 41 \\
\hline Latacunga & 6 & 10 \\
\hline & 58 & 100 \\
\hline
\end{tabular}

Fuente: Elaboración propia a partir de Encuesta de Agencias de Viajes Operadoras de Turismo (2017)

La credibilidad de la información se respalda en el criterio de los principales funcionarios de las operadoras de turismo siendo los gerentes propietarios, administradores o jefes de unidad quienes responden a la encuesta. En algunos casos quienes contestan las preguntas son los representantes legales por designación de los propietarios (ver tabla 2).

Tabla 2. Cargo que desempeñan los encuestados de las operadoras clasificados por ciudad

\begin{tabular}{|c|c|c|c|c|c|c|c|c|}
\hline \multirow[t]{2}{*}{ Ciudad } & \multicolumn{2}{|c|}{$\begin{array}{c}\text { Gerente } \\
\text { propietario }\end{array}$} & \multicolumn{2}{|c|}{ Administrador } & \multicolumn{2}{|c|}{$\begin{array}{l}\text { Jefe de } \\
\text { unidad }\end{array}$} & \multicolumn{2}{|c|}{ Otros } \\
\hline & $\#$ & $\%$ & $\#$ & $\%$ & \# & $\%$ & $\#$ & $\%$ \\
\hline Ambato & 5 & 23 & 0 & 0 & 3 & 75 & 4 & 22 \\
\hline Riobamba & 7 & 32 & 0 & 0 & 0 & 0 & 3 & 17 \\
\hline Puyo & 4 & 18 & 0 & 0 & 0 & 0 & 2 & 11 \\
\hline Baños & 3 & 14 & 13 & 93 & 1 & 25 & 7 & 39 \\
\hline \multirow[t]{2}{*}{ Latacunga } & 3 & 14 & 1 & 7 & 0 & 0 & 2 & 11 \\
\hline & 22 & 100 & 14 & 100 & 4 & 100 & 18 & 100 \\
\hline \multirow[t]{2}{*}{ Suman } & \multicolumn{8}{|c|}{58} \\
\hline & \multicolumn{2}{|c|}{$38 \%$} & \multicolumn{2}{|c|}{$24 \%$} & \multicolumn{2}{|c|}{$7 \%$} & \multicolumn{2}{|c|}{$31 \%$} \\
\hline
\end{tabular}

Entre los hallazgos más relevantes se encontró que el 91\% de las operadoras de la zona tres consideran muy importante la existencia de los guías nacionales de turismo para el desarrollo turístico en el Ecuador.

Tabla 3. Importancia de la existencia de guías nacionales clasificados por ciudad

\begin{tabular}{lccc}
\hline \multirow{2}{*}{ Ciudad } & \multicolumn{3}{c}{ Existencia de guías nacionales } \\
\cline { 2 - 4 } & $\begin{array}{c}\text { Muy } \\
\text { importante }\end{array}$ & $\begin{array}{c}\text { Poco } \\
\text { importante }\end{array}$ & $\begin{array}{c}\text { Nada } \\
\text { importante }\end{array}$ \\
\hline Ambato & 12 & 0 & 0 \\
\hline Riobamba & 8 & 2 & 0 \\
\hline Puyo & 6 & 0 & 0 \\
\hline Baños & 22 & 2 & 0 \\
\hline Latacunga & 5 & 0 & 1 \\
\hline Suma & 53 & 4 & 1 \\
\hline
\end{tabular}

Fuente: Elaboración propia a partir de Encuesta de Agencias de Viajes Operadoras de Turismo (2017)

En cuanto a los resultados obtenidos por parte de los guías de turismo, se obtuvo que el $56 \%$ de los entrevistados no poseen la credencial de guías nacionales y ejercen la actividad con otro tipo de carnets como lo es el de guía local o nativo, el carnet de guía naturalista y otro tipo de certificaciones (ver figura1). 


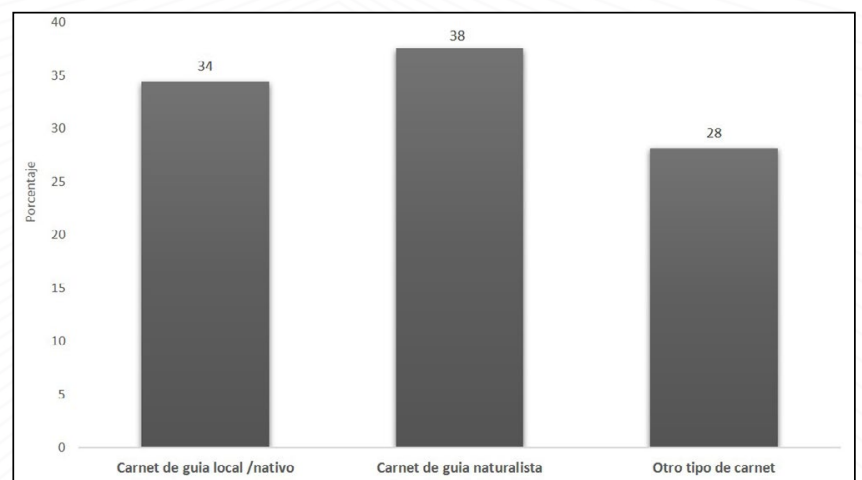

Fuente: Elaboración propia a partir de Encuesta a los Guías de Turismo de la zona 3 (2017) Figura 1. Utilización de carnets de guías de turismo

De igual forma se analizó con qué tipo de guías trabajan las operadoras, si son acreditados o no (ver figura2).

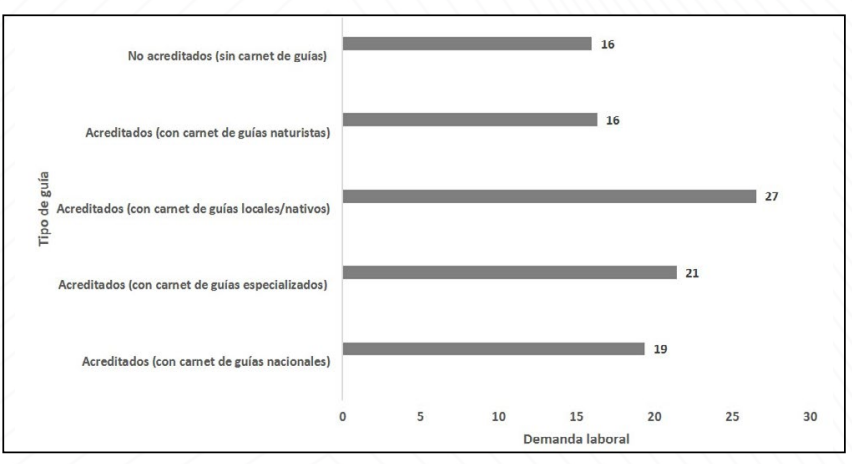

Fuente: Elaboración propia a partir de Encuesta a las Agencias de Viajes Operadoras de turismo (2017)

Figura 2. Demanda laboral según tipo de acreditación de guías

El $82 \%$ de las agencias de viajes ven como necesario que los guías de turismo tengan formación profesional. Sin embargo, el nivel de escolaridad que poseen los guías de turismo entrevistados esta entre titulación en el área de turismo, titulación en otras áreas y guías sin título profesional (ver figura3).

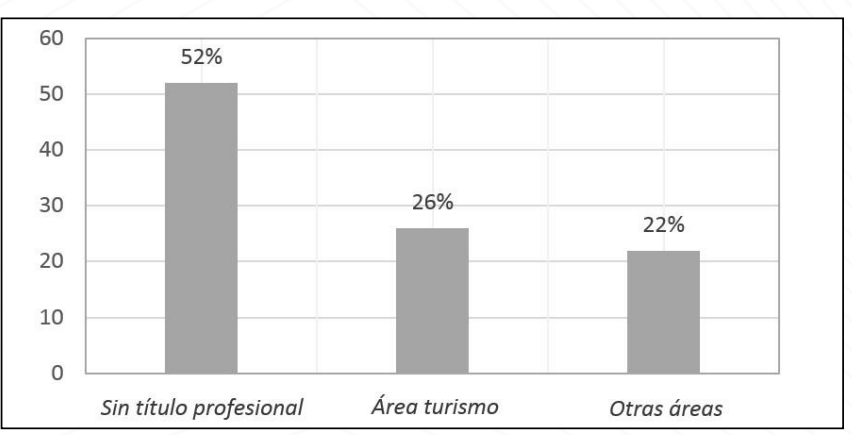

Fuente: Elaboración propia a partir de Encuesta a los Guías de Turismo de la zona 3 (2017) Figura 3. Titulación profesional

La necesidad de conocimiento es una variable importante, en la Tabla 3 , se puede identificar la priorización en el manejo de idiomas, conocimientos que le permiten al guía de turismo desarrollarse como promotor turístico, uno de los resultados establecidos en la Norma Técnica Ecuatoriana INEN 2 443:2007.
Tabla 4. Necesidad de conocimiento

\begin{tabular}{lrrr}
\hline Área & \multicolumn{3}{c}{ Nivel de importancia } \\
\hline Asistencia técnica & $50 \%$ & $75 \%$ & $100 \%$ \\
\hline Primeros auxilios & 7 & 20 & 19 \\
\hline Promotor turístico & 6 & 11 & 27 \\
\hline Animación de grupos & 7 & 7 & 32 \\
\hline Manejo de un segundo idioma & 10 & 8 & 28 \\
\hline Logística & 5 & 3 & 40 \\
\hline
\end{tabular}

Fuente: Elaboración propia a partir de Encuesta a los Guías de Turismo zona 3 (2017)

Las agencias de viajes consideran muy importante que existan guías nacionales para el desarrollo del turismo en el Ecuador. Puesto que son los que se encuentran en contacto directo con los turistas y por lo tanto influyen mucho en la imagen que ellos se llevan del país (Yu, Weiler, \& Ham, 2001). Sin embargo, no todos los guías turísticos poseen la credencial de guías nacionales tal como lo establece el Reglamento de Guianza Turística del Ecuador en el Art. 8, el cual menciona que para ser un Guía Nacional de Turismo acreditado debe como mínimo haber obtenido su título profesional en instituciones de educación superior. Los resultados reflejan una debilidad en la estructura de la oferta turística puesto que el $56 \%$ de los guías no poseen esta certificación.

Aquí surge una gran interrogante, ¿con qué tipo de documentación se brinda el servicio de guianza turística en la zona tres del país? Los resultados indican que lo realizan con carnets de guía local o nativo, carnet de guía naturalista y otro tipo de certificaciones. Este dato se contrasta con lo expresado por las agencias de viajes cuyos trabajadores en su mayoría poseen carnets de guías locales o nativos, carnets de guías especializados o naturalistas y carnets de guías nacionales, es decir si existe demanda de trabajo para los guías certificados.

En este estudio surge la admiración, al evidenciar que las agencias trabajan, aunque en un menor porcentaje, con guías sin ningún carnet o que simplemente no eligen ninguna opción. Es decir, existen agencias de viajes, aunque pocas que no cumplen a cabalidad con lo establecido en el artículo Art. 26 del Reglamento General de Actividades Turísticas del Ecuador, sobre la obligación en la contratación de guías nacionales de turismo, especializados, guías locales y tour leader en la ejecución de los diferentes programas turísticos. Cabe mencionar que los sectores donde se realizó el estudio, están más involucrados con excursiones cortas y el desarrollo de deportes extremos como es el caso del cantón Baños.

A pesar de existir un menor porcentaje de agencias que trabajan con guías sin carnet, el $82 \%$ es consciente de la necesidad de la formación profesional del guía. Sin embargo, la realidad de los guías turísticos es distinta puesto que apenas el $26 \%$ de los participantes se encuentran profesionalmente identificados en el área de turismo y el 56\% no poseen un título técnico o profesional. Este en el fondo podría llegar a ser un problema a la hora de brindar un servicio turístico de calidad. Tomando en cuenta que son los guías quienes han identificado las necesidades de conocimiento que deben desarrollar para ejercer la profesión y resaltan el manejo de un segundo idioma, el poder comunicarse en más de un idioma se vuelve una herramienta competitiva para los guías y esto genera mayor demanda (Zárraga, Corona, \& Molina, 2010). Además, se menciona otros conocimientos como la promoción turista, la animación de grupos, conocer sobre primeros auxilios, logística y la asistencia técnica.

Donde hace falta capacitar a los guías es en primeros auxilios, este conocimiento es valorado tanto por los tour operadoras como por los propios guías de turistas (Zárraga, Corona, \& Molina, 2010). El análisis de los ámbitos de conocimiento, van acompañados adicionalmente con el desenvolvimiento de las competencias laborales que tienen que ver con la organización, procedimientos administrativos, orientación y 
apoyo, calidad en el servicio, asegurar el bienestar del turista, manejo de grupos, y promover el turismo INEN (2 443:2007), es decir, quien se desempeña como Guía Nacional de Turismo abarca una extensa gama de conocimientos y habilidades, lo que le conduce a una amplia formación profesional.

Las tour operadoras buscan constantemente guías de turismo que tengan una preparación profesional de tercer nivel (Zárraga, Corona, \& Molina, 2010). Los títulos de grado y postgrado en turismo deben ir orientados a las necesidades del sector. De esta manera se puede lograr un mayor acercamiento del ámbito académico con el sector público y privado para establecer sinergias que contribuyan a mejorar el capital humano y la investigación del turismo en el país (Bañuls, Rodríguez, \& Jiménez, 2007). Porque es en los guías de turismo en quienes recae la función de dar a conocer el patrimonio cultural, natural y gastronómico de un destino. Son ellos quienes tiene el primer contacto con los turistas y asumen la responsabilidad de transmitir no solo una información precisa y útil, sino la imagen del destino, además de promover su atractivo turístico (Ramallo, 2015).

\section{Conclusiones}

La zona 3 del Ecuador, se considera como un punto estratégico de confluencia en la región, en tal motivo el estudio de la oferta y demanda de los guías de turismo se ha concentrado específicamente en este sector. Durante la investigación se ha podido determinar que las agencias de servicios turísticos como intermediadoras entre la oferta y demanda turísticas en un $91 \%$ están muy consientes sobre la importancia del rol que cumplen los guías profesionales, en el Ecuador, aunque existe un 9\% que no piensa de esta manera, esto es preocupante puesto que no le dan importancia al trabajar con guias certificados, perjudicando a los profesionales del turismo y a los turistas. Esta es una tarea pendiente a ser cosiderada por las autoridades que regulan la actividad turística. Pese al reducido porcentaje de agencias turísticas que no trabajan con guías certificados le sigue otro porcentaje del $28 \%$ de guías de turismo que ejercen sus actividades con otro tipo de carné que no corresponden a los autorizados por el Ministerio de Turismo del Ecuador. Lo que se demuestra con el anterior resultado es que se subestima el rol que juega el guía de turismo tanto en la programación como en la ejecución de tours. Esto se realiza con la anuencia de los propietarios de las agencias de viaje, se subestima el rol que juega el guía de turismo tanto en la programación como en la ejecución de tours, permitiéndoles operar y trabajar, aunque no posean la certificación reglamentaria, por este motivo los guías no se preocupan por legalizar su situación laboral. La remuneración que perciben en estos casos es menor.

Este análisis se contrapone a la realidad lo que se refleja en el $16 \%$ de las operadoras de turismo encuestadas que no trabajan con guías cualificados, es decir, se pone en riesgo el factor de percepción del turista que transformado en términos de calidad ese porcentaje podría afectar notablemente el índice de satisfacción del turista si se toma en cuenta, que en términos de calidad, el $20 \%$ de clientes insatisfechos puede convertirse en el $50 \%$. Se debe concientizar a los propietarios de las agencias de viajes para que en su totalidad dispongan de personal calificado para ejercer la profesión, como se establece en el Art. 26 del Reglamento General de Actividades Turísticas del Ecuador en torno al tema de contratación de guías acreditados y en el Reglamento de Guianza Turística del Ecuador donde se especifíca claramente que un guía nacional de turismo acreditado debe tener como mínimo una titulación profesional obtenida en instituciones de educación superior. Esto garantizaría una mejora en la calidad de atención al turista, tanto local como internacional.

En la investigación también se ha tomado en cuenta otro aspecto como es el nivel de titulación que poseen los guías de turismo, puesto que se pudo constatar que apenas el $26 \%$ de los guías encuestados son especialistas en el área; en cambio, el 22\% posee título de tercer nivel pero en campos de conocimientos diferentes al área ocupacional en la que se desempeñan. Estos resultados reflejan el desinterés que le dan las agencias de viajes a la hora de contar con profesionales que cubran el perfil requerido para cada uno de los segmentos turísticos de la demanda turística. Lo anterior deja abierta la oportunidad para que los profesionales del turismo ocupen esas plazas de trabajo, siempre y cuando las operadoras deseen mejorar su calidad en el servicio al cliente.

Las especificaciones detalladas en la norma técnica ecuatoriana indican que el guía de turismo debe dar respuesta a varios procedimientos como: logística, asistencia de varios tipos, promotor turístico, entre otros. Así mismo, lo que se pudo identificar en el estudio es que el idioma que predomina en quienes trabajan como guías es el inglés, seguido del alemán, francés e italiano; lo que demuestra a la vez, es que se amplía el portal de conocimientos, aptitudes y destrezas enmarcadas dentro de la gran gama de competencias laborales que debe poseer el guía de turismo, convirtiéndose en su fortaleza más importante al momento de trabajar con turistas extranjeros.

De estos resultados se puede afirmar que al contratar personal sin la certificación correspondiente no se puede garantizar que la experiencia del turista en el país haya sido placentera, pues para llegar a ese nivel realmente se requiere del compromiso aunado tanto de la empresa privada como de las políticas estatales.

\section{Referencias}

AENOR. (10 de 02 de 2016). aenor.es. Obtenido de http://www.aenor.es/ aenor/normas/normas/fichanorma.asp?tipo=N\&codigo=N0056174\#.WSdAtWiGPIV Bañuls, A. L., Rodríguez, A. B., \& Jiménez, M. S. (2007). El capital humano como factor estratégico para la competitividad del sector turístico. Cuadernos de turismo, 19, 47-69.

Bote, V. (1995). Estructura y desarrollo del turismo en España: hacia un cambio cualitativo y más responsable. España: In Congreso de Economía del Turismo.

Boullón, R. (2006). El Sistema Turístico. México: TRILLAS.

Cohen, E. (1985). The tourist guide: The origins, structure and dynamics of a role. 1985 The tourist guide: The origins, structure and dynamics of a role. Annals of Tourism Research , 12(1), 5-29.

Ham, S. H., \& Weiler, B. (2003). Capacitación para guías turísticos en Latinoamérica. Estudios y Perspectivas en Turismo, 12, 24-47.

Huizinga, J. (2007). Homo Ludens. Madrid : Alianza.

INEC. (2017). Encuesta Nacional de empleo, desempleo y subempleo. Indicadores laborales. Quito: INEC.

ISO. (2007). Norma ISO para guías de turismo. Quito, Pichincha.

López, M. (2013). Aprendizaje, competencias y TIC. México: PEARSON. MINTUR. (2017d). Listado de los Principales Destinos del Ecuador para Turismo Receptivo. 2017. Quito: MINTUR- Dirección de productos y destinos 2017. MINTUR. (26 de Abril de 2017b). Ministerio de Turismo. Obtenido de En-

tradas y Salidas Internacionales por Nacionalidad: $h$ ttp://servicios.turismo.gob.ec MINTUR. (03 de diciembre de 2017a). Ministerio de Turismo. Obtenido de No-

ticias: https://uww.turismo.gob.ec/arnibo-de-turistas-a-ecuador-crecio-en-14-en-2017/ MINTUR. (05 de junio de 2017c). Ministerio de Turismo . Obtenido de Noticias: https://www.turismo.gob.ec/el-turismo-sera-el-eje-del-cambio-de-la-matrizproductiva-en-ecuador/

OMT. (2016). Panorama OMT del Turismo Internacional. Edición 2016. UNWTO. OMT. (2017). Panorama OMT del Turismo internacional. Edición 2017. UNWTO OMT; UNESCO. (2015). La Conferencia Mundial sobre Turismo y Cultura. Camboya: OMT, UNESCO

Pond, K. (1993). The professional guide: dynamics of tour guiding.

New York: Van Nostrand Reinhold.

Prado, V. S. (2001). Oportunidades de capacitación de recursos humanos en turismo. Santiago: Servicio Nacional de Turismo .

Ramallo, E. (2015). EL GUIIA TURÍSTICO DIFERENCIAS Y SIMILITUDES

ENTRE COMUNIDADES AUTÓNOMAS. Cuadernos de Turiso(35), 499-510.

Rebolledo Dujisin, P., \& Fica Pérez, R. (2010). La formación de guías de 
turismo de montaña y alta montaña: el caso chileno. Gest. tur.(Valdivia), 14, 83-95. Reglamento de Guianza Turística. (2016). Quito: Lexis.

Reglamento General de Actividades Turísticas. (2011). Quito: Lexis. Swarbrooke, J. (1999). Sustainable Tourism Management. UK: CABI Publishing.

Yu, X., Weiler, B., \& Ham, S. (2001). Intercultural communication and mediation: A framework for analysing the intercultural competence of Chinese tour guides. Journal of Vacation Marketing, 2 - 3.

Zárraga, L., Corona, E., \& Molina, V. (2010). Las competencias de los guias de turistas como ventaja competitiva en el sector turista: Caso Cancun Quintana Roo. REVISTA INTERNACIONAL ADMINISTRACION \& FINANZAS, 3(3), 11-24.

Anexos páginas 24 y 25 